\title{
Mini-Review
}

\section{Towards a Genetic Definition of Cancer-Associated Inflammation}

\section{Role of the IDO Pathway}

\author{
George C. Prendergast, ${ }^{\star \dagger \ddagger}$ Richard Metz, ${ }^{\S}$ \\ and Alexander J. Muller ${ }^{\star \ddagger}$ \\ From the Lankenau Institute for Medical Research," Wynnewood, \\ Pennsylvania; the Department of Pathology, Anatomy, and Cell \\ Biology, Jefferson Medical School, and Kimmel Cancer Center, ${ }^{\dagger}$ \\ Thomas Jefferson University, Philadelphia, Pennsylvania; and \\ New Link Genetics Corporation, ${ }^{\Im}$ Ames, Iowa
}

Chronic inflammation drives the development of many cancers, but a genetic definition of what constitutes 'cancer-associated' inflammation has not been determined. Recently, a mouse genetic study revealed a critical role for the immune escape mediator indoleamine 2,3-dioxygenase (IDO) in supporting inflammatory skin carcinogenesis. IDO is generally regarded as being immunosuppressive; however, there was no discernable difference in generalized inflammatory processes in IDO-null mice under conditions where tumor development was significantly suppressed, implicating IDO as key to establishing the pathogenic state of 'cancer-associated' inflammation. Here we review recent findings and their potential implications to understanding the relationship between immune escape and inflammation in cancer. Briefly, we propose that genetic pathways of immune escape in cancer are synonymous with pathways that define 'cancer-associated' inflammation and that these processes may be identical rather than distinct, as generally presumed, in terms of their genetic definition. (Am J Pathol 2010, 176:2082-2087; DOI: 10.2353/ajpath.2010.091173)

\section{Background: Inflammation and Immune Escape in Cancer Progression}

Cancer is initiated by cumulative genetic and epigenetic changes to a normal cell. However, beyond the cancer cell itself, it is clear that the tissue microenvironment exerts powerful effects in determining progression versus dormancy or destruction of an oncogenically-initiated cell. Thus, while oncogenesis initiates cancer, modifier effects exerted by the tissue microenvironment guide progression. Indeed, loss of microenvironmental control may be essential to unleash invasive and metastatic behaviors that pose the greatest clinical challenge. A wide variety of immune cells in the inflammatory microenvironment can contribute powerfully to tumor suppression. Yet it is also evident that tumors can evolve tactics to 'tilt' the immune balance from antagonistic to supportive. Although an appropriately activated immune system can eradicate cancer in an animal, even when widely disseminated, it is clear that cancers erect barricades to overcome the awesome power of the immune system. Thus, the balance between immunosuppressive and immunostimulatory signals in the inflammatory microenvironment has profound implications for tumor eradication, control (dormancy), or outgrowth.

Chronic inflammation is a key component in the genesis and progression of many solid tumors. The earliest descriptions of cancer histopathology in the 1800s by Virchow and others noted striking hallmarks of inflammation. ${ }^{1}$ Despite the strong evidence linking inflammation and cancer, there remains a pressing need to define the specific factors that define 'cancer-associated' inflammation as it is distinguished from other contexts of chronic or classical

Supported by grants from the National Cancer Institute, DoD Breast Cancer Research Program, Lance Armstrong Foundation, American Lung Association, Dan Green Foundation, New Link Genetics Corporation, Lankenau Hospital Foundation, and the Main Line Health System.

Accepted for publication January 19, 2010

G.C.P. and A.J.M. declare a conflict of interest with regard to IDO inhibitor studies due to intellectual property, financial interests, grant support, and consultancy roles with New Link Genetics Corporation, which is engaged in clinical development of IDO inhibitors for the purpose of treating cancer and other diseases.

A guest editor acted as editor-in-chief for this manuscript. No person at Thomas Jefferson University was involved in the peer review process or final disposition for this article.

Address reprint requests to George C. Prendergast, Ph.D., Lankenau Institute for Medical Research, 100 Lancaster Avenue, Wynnewood PA 19096. E-mail: prendergast@limr.org 


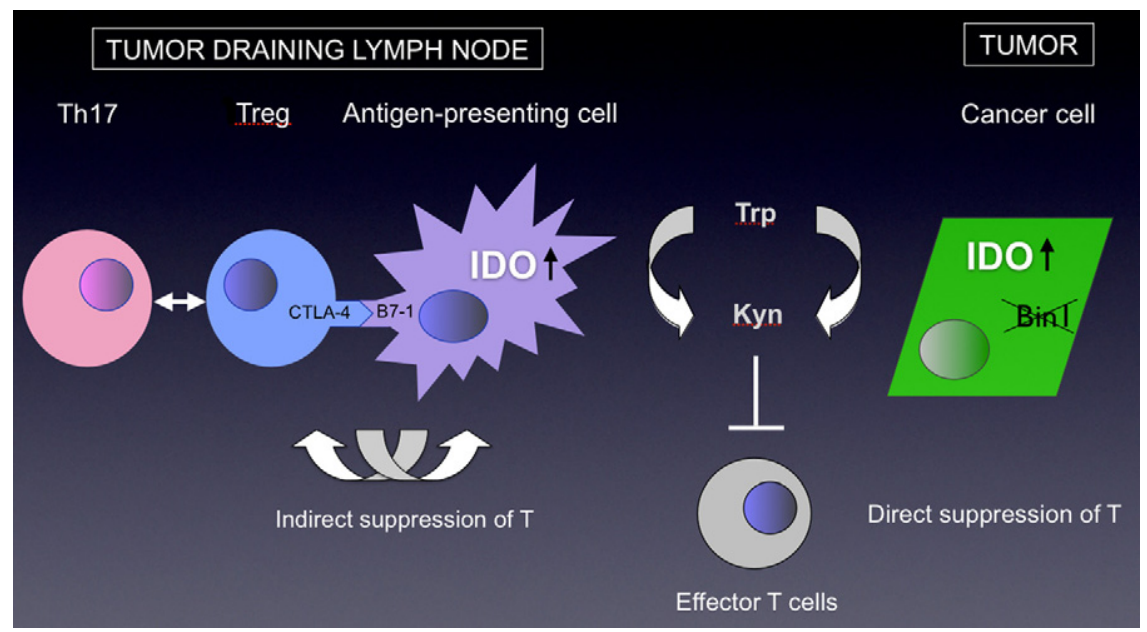

Figure 1. Dual roles of IDO in cancer. IDO is overexpressed in many types of tumor cells where its presence has been associated with poor prognosis. ${ }^{6}$ In tumor cells, IDO dysregulation has been linked to inactivation of tumor suppressor gene Bin1, an amphiphysin-like gene that is widely attenuated or misspliced in cancer. ${ }^{13,14}$ In tumor-draining lymph nodes, IDO is upregulated in a regulatory subset of plasmacytoid dendritic cells that has been suggested to inform a positive feedback loop in the activation of $\mathrm{T}$ regulatory cells (Tregs), while also blunting their interconversion to Th17 inflammatory cells. ${ }^{15,16}$ IDO activity consumes tryptophan, depriving it from local tissue microenvironments, also generating kynurenine as a product. Both of these processes have been implicated in $\mathrm{T}$ cell suppression, including by blunting $\mathrm{T}$ effector cell function as well as recruitment of Tregs. ${ }^{12}$

inflammatory processes that are not associated with cancer. ${ }^{2}$ The explanative impact of answers to this question would be strengthened by their ability to shed light on how 'cancer-associated' inflammation is integrated with the processes of oncogenesis and immunoediting.

As introduced by Schreiber, Smyth, and their colleagues, immunoediting suggests a description of how the thrust and parry of the battle between the tumor and the immune system gradually leads to a loss of immune control of neoplastic cells. ${ }^{3}$ Immunoediting has three stages that represent a conceptual veneer atop more widely known and accepted models of oncogenesis. The first stage of immunoediting corresponds to immune surveillance as enunciated originally by Ehrlich and later by Burnett and Lewis. Tumor neoantigens and loss of self arising from oncogenesis can be recognized by the immune system, leading to tumor cell eradication. However, the selective pressure imposed by immune surveillance facilitates evolution in the genetically plastic tumor cell toward immune equilibrium. At this second stage, the immune system can control a tumor but not fully eradicate it (similar to a low-grade infection). Immune equilibrium may explain the clinical phenomena of tumor dormancy, which can occur over long periods. Recently, direct evidence of immune equilibrium has been offered as a mechanism to explain tumor dormancy in mice. ${ }^{4}$ By iterating resistance mechanisms, tumor cells can 'sculpt' the immune system beyond equilibrium to achieve the final stage of immune escape. Achieving this fundamental hallmark of cancer signifies the total defeat of the immune system in tumor suppression. Although not all model systems may fit this rule, ${ }^{5}$ iterating immune escape mechanisms could allow cancer cells to ultimately 'tilt' immunity from tumor eradication to tumor tolerance.

\section{IDO in Immune Tolerance and Escape}

The tryptophan catabolic enzyme indoleamine 2,3-dioxygenase-1 (IDO) mediates immune tolerance in a variety of physiological and pathological settings, including cancer. ${ }^{6}$ This single chain oxidoreductase was initially discovered by its ability to catalyze tryptophan degradation to the first step in biosynthesis of nicotinamide adenine dinucleotide IDO does not handle dietary tryptophan catabolism, and nicotinamide adenine dinucleotidelevels in mammals are maintained by salvage rather than synthesis. Thus, the role of IDO in mammalian cells was generally unclear before seminal studies of Munn, Mellor, and colleagues, who correlated IDO activity with $\mathrm{T}$ cell suppression. ${ }^{7,8}$ IDO can be expressed in a variety of cell types including antigen-presenting cells in lymph nodes, where an immunoregulatory function has been suggested. ${ }^{9}$ Through local tryptophan starvation, IDO can stimulate a Gcn2-induced stress signaling pathway ${ }^{10}$ that upregulates translation of the NF-IL6/CEBP $\beta$ transcription factor isoform LIP, which alters immune-related gene expression including the key immune modulatory factors interleukin-6, transforming growth factor- $\beta$, and interleukin-10. ${ }^{11}$ In a dramatic illustration of how IDO can mediate local immunosuppression to neoantigens, the bioactive IDO inhibitor 1-methyl-tryptophan (1MT) will trigger major histocompatibility complex-restricted T cell-mediated rejection of allogeneic pregnancies by revealing 'foreign' paternal antigens to the maternal immune system. ${ }^{7}$

Strikingly, IDO is widely dysregulated in tumors and tumor-draining lymph nodes (TDLNs), where it has been implicated in facilitating immune escape. ${ }^{12}$ Figure $1^{5,12-16}$ summarizes literature on dual roles of IDO in cancer as related to its dysregulation in tumors or TDLNs. Genetic studies in the mouse revealed that IDO is suppressed by the tumor suppressor gene Bin1, which is often attenuated in human cancers. ${ }^{13,14}$ Bin1 also limits the IFN- $\gamma$ mediated activation of IDO in myeloid cells. ${ }^{13}$ Moreover, $1 \mathrm{MT}$ and other structural classes of IDO inhibitors identified in our laboratory can correct IDO-mediated immune escape, stanching tumor growth and synergizing with chemotherapy to eradicate established tumors in a variety of transgenic and allograft mouse models of cancer (in a T cell-dependent manner). Recent experiments in IDO-deficient mice confirm that IDO must be present for $1 \mathrm{MT}$ or other inhibitors to manifest their antitumor effects. ${ }^{17-20}$ 
In cancer, IDO may be overexpressed in tumor cells, TDLNs, or both. 9,13,21,22 In TDLNs, specific subsets of antigen presenting cells appear to be preferentially capable of expressing IDO. In mice, these 'IDO-competent' antigen presenting cells include a subset of plasmacytoid dendritic cells (pDCs) distinguished by the cell surface markers B220 and CD19 (typically associated with the B cell lineage), which have been implicated in supporting Treg development and malignant outgrowth. 23,24 Interestingly, IDO induction in these pDCs appears to be sufficient to convert them from a $T$ cell-activating to $T$ cell-suppressing phenotype. Dermal application of a proinflammatory phorbol ester induces IDO+ pDCs that potently suppress antigen-dependent $\mathrm{T}$ cell activation. ${ }^{20}$ In addition to directly suppressing T cell activation, IDO+ pDCs have also been reported to inform the organization of a population of $\mathrm{T}$ regulatory cells (Tregs) by inducing naïve $\mathrm{CD} 4^{+} \mathrm{T}$ cells to differentiate to FoxP3+ Tregs, as well as by activating resting natural Tregs to become potently suppressive. ${ }^{24,25}$ Interestingly, Tregs have been shown to induce IDO in DCs through CTLA-4-mediated reverse signaling. ${ }^{26-28}$ Therefore, it has been suggested that communication between IDO+ pDCs and Tregs may inform a positive feedback loop of immunosuppression that helps drive cancer progression. As an initial step in efforts to therapeutically disable this pathway in cancer, the $D$ isomer of $1 \mathrm{MT}$ (D-1MT) has progressed to Phase I human studies, with some early promising signs that its oral administration can yield biological activity at safely tolerated doses permitting further clinical evaluation. ${ }^{29}$

\section{IDO is Essential for Inflammatory Skin Carcinogenesis}

In skin, where IDO connects chronic inflammation to the production of a potent immune suppressive class of IDO + pDCs, we hypothesized that IDO would also connect chronic inflammation to cancer progression, where immune escape may be pivotal. ${ }^{4,12,30}$ If IDO had a generalized immunosuppressive role in inflammation, its deficiency in mice might alter the course of normal wound healing or the epidermal thickening that occurs after application of including phorbol esters. Alternately, if IDO had a more subtle role in contextualizing the inflammatory microenvironment in a manner that was apparent only in certain settings, such as cancer development, then IDO deficiency in mice would not be expected to affect general processes of inflammation. Indeed, no differences were noted in the histological response of IDO-deficient mice to abrasion wounds or topical treatment with a proinflammatory phorbol ester. However, within the same time frame, IDO was critical for the generation of immune suppressive IDO+ pDCs appearing in inflamed skindraining LNs after phorbol ester treatment. ${ }^{20}$ These observations suggested that IDO contributes to a specific or peculiar aspect of inflammation rather than overtly impacting its progression. Strikingly, this aspect of the inflammatory response to phorbol ester was associated with a deficiency in the formation and malignant progression of skin tumors (papillomas and carcinomas). ${ }^{20}$
A

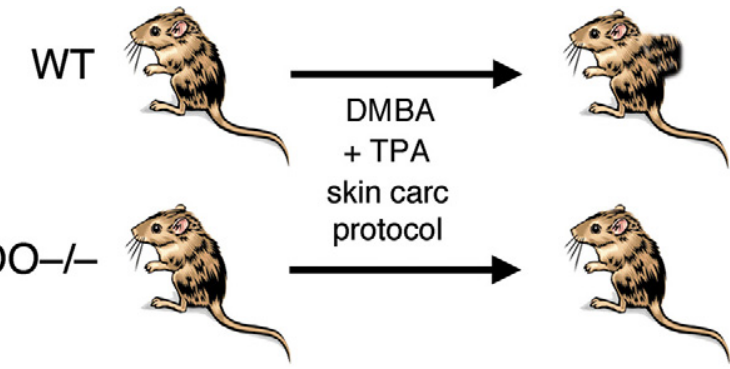

B

Traditional Perspective

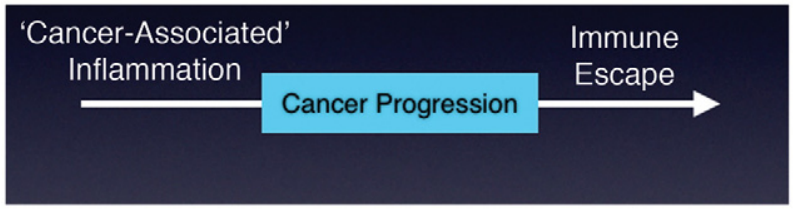

Proposed Perspective

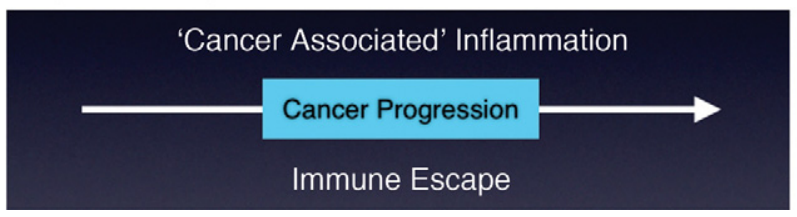

Figure 2. Genetic overlap between immune escape and 'cancer-associated' inflammation. A: IDO-deficient mice are resistant to inflammatory skin carcinogenesis. Panels compare several representative $\mathrm{IDO}^{+/+}$and $\mathrm{IDO}^{-1}$ mice from cohorts subjected to a standard classical protocol of two-stage inflammatory skin carcinogenesis that involves a single topical application of DMBA followed by chronic exposure to phorbol ester TPA. Figure derived from Muller et $\mathrm{al}^{20}$ with permission of the Proceedings of the National Academies of Sciences of the United States of America. B: Traditional versus proposed perspectives on the relationship between 'cancer-associated' inflammation and immune escape in the tumor microenvironment. The arrows represent time. Top, The inflammatory tumor microenvironment precedes and engenders immune 'sculpting' that supports the development of immune escape. Bottom, The peculiar cancer-supporting elements of the inflammatory tumor microenvironment are genetically identical to the elements that support immune escape.

Briefly, the susceptibility of IDO-null mice to inflammatory skin carcinogenesis was compared with genetically identical wild-type mice using a classical multistage protocol of initiation by carcinogen 7,12-dimethylbenz[a]anthracene (DMBA) followed by promotion with twice weekly application of the phorbol ester 12-O-tetradecanylphorbol-13-acetate (TPA). Although we observed no difference in IDO-null mice with regard to the time of onset of skin papillomas, which arose at 8 to 10 weeks after initiation as expected, IDO-null mice exhibited a greatly reduced incidence of papilloma formation, with significantly fewer lesions arising over the 20-week course of TPA treatment. ${ }^{20}$ Continued monitoring beyond 20 weeks permitted efficient conversion to skin carcinoma in wild-type mice but not in IDO-deficient mice, with the wild-type mice exhibiting tumors that were also more advanced and obviously transformed than those of their IDO-null counterparts (Figure 2A). ${ }^{20}$ Thus, IDO was critical in this classical model of carcinogenesis driven by chronic inflammation, both at the level of tumor formation as well as tumor progression. Because IDO-deficient mice can support the growth of primary tumors formed by engrafted cancer cell lines of various types, it is clearly not required 
for tumor growth per se but instead may facilitate cancer development as a mediator of 'cancer-associated' inflammation. Indeed, IDO may offer a defining element to understand the nature of this peculiar pathological condition.

\section{Implications of IDO in Defining 'Cancer-Associated' Inflammation}

The molecular factors that define chronic 'cancer-associated' inflammation have been unclear. Inflammation can be caused by infection, tissue damage, or both. After infection, inflammation activates innate immune functions and enhances immune cell infiltration into affected tissues and proximal lymph nodes. After tissue damage, 'alarmins' or other 'danger-associated' signals released by dying cells in the absence of infection can also provoke inflammation. In chronic infections or chronic tissue damage, inflammatory responses that stimulate effective $T$ cell immunity and memory appear to be blunted through the generation of active states of T cell suppression. By analogy, chronic inflammatory responses associated with the development and progression of cancer may be similar to those associated with chronic infection, where functions like IDO that can suppress T cell immunity and memory are invoked.

Neoantigens and cell death that arise during tumorigenesis can each provoke inflammation, but the molecular mechanisms that permit tumor cells to persist in immunocompetent individuals remain obscure. What is clear is that local inflammation provides growth factors and cytokines that help sustain the growth deregulation, survival, and movement of premalignant cells. ${ }^{31,32}$ Additionally, inflammation can facilitate tumor progression by creating microenvironments at both the primary site of the tumor as well as the TDLNs that restrict immune surveillance. 3,4,33,34 Active suppression of adaptive immunity obviously occurs, because by the time tumors are overtly manifested both the tumor microenvironment and the TDLNs harbor potent T cell suppressor functions. ${ }^{35-37}$ Thus, in considering how tumor cells acquire the ability to exploit inflammation and subvert immune surveillance, it is clear that they retain supportive features of the inflammatory microenvironment as tactics evolve to escape features hostile to the tumor. Thus, mechanisms that integrate immune escape with chronic inflammation would be predicted to promote carcinogenesis.

IDO may provide a functional link between immune escape and chronic inflammatory states that drives the development and progression of many cancers. In providing a defining element of 'cancer-associated' inflammation, this connection has conceptual and practical implications.

One implication is that the genetic mechanisms underpinning immune escape and 'cancer-associated' inflammation may be overlapping rather than distinct (Figure 2B). Models suggesting how the inflammatory microenvironment contributes to cancer tend to be based on the notion that the inflammatory conditions precede and engender the later evolution of immune escape mecha- nisms. However, studies in the IDO-deficient mouse argue that this concept may be invalid, because a single gene that supports immune escape is also needed at early times to support the generation of a 'cancer-associated' inflammatory state needed for tumorigenesis. If immune escape pathways are understood merely as pathogenic elements of inflammation needed to support common cancers-a 'molecular flavor' - then 'cancer-associated' inflammation might be defined as a 'general' chronic inflammation plus a genetic or epigenetic signature of immune escape, such as chronic IDO activation.

A second implication is that immune escape may be initiated early in cancer, rather than later during tumor progression as the standard model of immunoediting proposes. This concept impacts other skepticisms raised to immunoediting, which at root question the idea that immune escape is the end result of an evolutionary process of immune 'sculpting' by tumor-microenvironment interplay, rather than an aberration of the immune system that is fixed at early times in tumor formation. ${ }^{5,38}$

A third implication concerns the common use of mouse xenograft models to study cancer development and treatment. The preclinical development of most cancer drugs uses such models, which involve the engraftment of human tumor cells into immune compromised animals. In fact, these models remain an industry standard to evaluate traditional cytotoxics as well as modern molecular targeted drugs, rooted in seminal work done in immunodeficient nude mice by Rygaard and Povlsen more than 40 years ago. ${ }^{39}$ Nude mice do not have higher spontaneous rates of spontaneous cancer, which suggested to many at the time that immunity was nonessential in tumor suppression. However, while setting a tone that later helped push tumor immunology away from the mainstream of cancer research, tumor suppressive natural killer cells, which are intact in nude mice were unknown until later, ${ }^{40}$ and a convincing genetic refutation of the skepticism about immunity as an essential tumor suppression function was not achieved until this century by Schreiber and colleagues. ${ }^{30}$ With the more recent emergence of similar proofs of the great significance of immune-mediated inflammatory processes to oncogenesis and progression, ${ }^{41}$ such as processes supported by IDO, ${ }^{20}$ already existing concerns about the pathophysiological relevance of mouse xenograft models to clinical oncology are heightened still further.

One final implication concerns tactics to defeat or correct immune escape in cancer, which might be understood more simply as a reprogramming of the inflammatory state of the tumor microenvironment, altering its 'molecular flavor' to antagonize rather than nurture the tumor. With regard to IDO inhibitors being explored for cancer therapy, this notion is intriguing in light of recent evidence that IDO blockade can promote the conversion of tumor-associated Tregs to Th17-like effector T cells, which might stimulate an antitumor response. ${ }^{15,16}$ The notion of therapeutic impact from specific inhibitors of pathogenic components of inflammation that support tumoral immune escape may represent a focused approach to cancer, ${ }^{42}$ relative to generalized anti-inflammatory approaches that as a broader brush have garnered 
significant attention. As we first suggested some time ago, ${ }^{43}$ investigations to connect immune escape mechanisms with 'cancer-associated' inflammation will further develop these concepts and their prognostic or therapeutic applications.

\section{Acknowledgment}

We apologize to scientific colleagues whose work could not be fully cited because of space limitations.

\section{References}

1. Balkwill F, Mantovani A: Inflammation and cancer: back to Virchow? Lancet 2001, 357:539-545

2. Peek RM, Mohla S, DuBois RN: Inflammation in the genesis and perpetuation of cancer: summary and recommendations from a National Cancer Institute-sponsored meeting. Cancer Res 2005, 65:8583-8586

3. Dunn GP, Old LJ, Schreiber RD: The immunobiology of cancer immunosurveillance and immunoediting. Immunity 2004, 21:137-148

4. Koebel CM, Vermi W, Swann JB, Zerafa N, Rodig SJ, Old LJ, Smyth MJ, Schreiber RD: Adaptive immunity maintains occult cancer in an equilibrium state. Nature 2007, 450:903-907

5. Willimsky G, Blankenstein T: The adaptive immune response to sporadic cancer. Immunol Rev 2007, 220:102-112

6. Katz JB, Muller AJ, Metz R, Prendergast GC: Indoleamine 2,3-dioxygenase in T-cell tolerance and tumoral immune escape. Immunol Rev 2008, 222:206-221

7. Munn DH, Zhou M, Attwood JT, Bondarev I, Conway SJ, Marshall B, Brown C, Mellor AL: Prevention of allogeneic fetal rejection by tryptophan catabolism. Science 1998, 281:1191-1193

8. Munn DH, Shafizadeh E, Attwood JT, Bondarev I, Pashine A, Mellor AL: Inhibition of T cell proliferation by macrophage tryptophan catabolism. J Exp Med 1999, 189:1363-1372

9. Munn DH, Sharma MD, Hou D, Baban B, Lee JR, Antonia SJ, Messina JL, Chandler P, Koni PA, Mellor AL: Expression of indoleamine 2,3dioxygenase by plasmacytoid dendritic cells in tumor-draining lymph nodes. J Clin Invest 2004, 114:280-290

10. Munn DH, Sharma MD, Baban B, Harding HP, Zhang Y, Ron D, Mellor AL: GCN2 kinase in T cells mediates proliferative arrest and anergy induction in response to indoleamine 2,3-dioxygenase. Immunity 2005, 22:633-642

11. Metz R, DuHadaway JB, Kamasani U, Laury-Kleintop L, Muller AJ, Prendergast GC: Novel tryptophan catabolic enzyme IDO2 is the preferred biochemical target of the antitumor IDO inhibitory compound D-1MT. Cancer Res 2007, 67:7082-7087

12. Prendergast GC: Immune escape as a fundamental trait of cancer: focus on IDO. Oncogene 2008, 27:3889-3900

13. Muller AJ, DuHadaway JB, Sutanto-Ward E, Donover PS, Prendergast GC: Inhibition of indoleamine 2,3-dioxygenase, an immunomodulatory target of the tumor suppressor gene Bin1, potentiates cancer chemotherapy. Nat Med 2005, 11:312-319

14. Prendergast GC, Muller AJ, Ramalingam A, Chang MY: BAR the door: cancer suppression by amphiphysin-like genes. Biochim Biophys Acta 2009, 1795:25-36

15. Baban B, Chandler PR, Sharma MD, Pihkala J, Koni PA, Munn DH, Mellor AL: IDO activates regulatory $T$ cells and blocks their conversion into Th17-like T cells. J Immunol 2009, 183:2475-2483

16. Sharma MD, Hou DY, Liu Y, Koni PA, Metz R, Chandler P, Mellor AL, He $Y$, Munn DH: Indoleamine 2,3-dioxygenase controls conversion of Foxp3+ Tregs to TH17-like cells in tumor-draining lymph nodes. Blood 2009, 113:6102-6111

17. Hou DY, Muller AJ, Sharma MD, DuHadaway J, Banerjee T, Johnson M, Mellor AL, Prendergast GC, Munn DH: Inhibition of indoleamine 2,3dioxygenase in dendritic cells by stereoisomers of 1-methyl-tryptophan correlates with antitumor responses. Cancer Res 2007, 67:792-801

18. Banerjee T, DuHadaway JB, Gaspari P, Sutanto-Ward E, Munn DH, Mellor AL, Malachowski WP, Prendergast GC, Muller AJ: Antitumor properties of chemopreventive natural product brassinin are based upon inhibition of indoleamine 2,3-dioxygenase (IDO). Oncogene 2008, 27:2851-2857

19. Kumar S, Malachowski WP, DuHadaway JB, LaLonde JM, Carroll PJ, Jaller D, Metz R, Prendergast GC, Muller AJ: Indoleamine 2,3-dioxygenase is the anticancer target for a novel series of potent naphthoquinone-based inhibitors. J Med Chem 2008, 51:1706-1718

20. Muller AJ, Sharma MD, Chandler PR, Duhadaway JB, Everhart ME Johnson BA 3rd, Kahler DJ, Pihkala J, Soler AP, Munn DH, Prendergast GC, Mellor AL: Chronic inflammation that facilitates tumor progression creates local immune suppression by inducing indoleamine 2,3 dioxygenase. Proc Natl Acad Sci USA 2008, 105:17073-17078

21. Uyttenhove C, Pilotte L, Theate I, Stroobant V, Colau D, Parmentier N, Boon T, Van Den Eynde BJ: Evidence for a tumoral immune resistance mechanism based on tryptophan degradation by indoleamine 2,3-dioxygenase. Nat Med 2003, 9:1269-1274

22. Munn DH, Mellor AL: Indoleamine 2,3-dioxygenase and tumor-induced tolerance. J Clin Invest 2007, 117:1147-1154

23. Munn DH: Indoleamine 2,3-dioxygenase, tumor-induced tolerance and counter-regulation. Curr Opin Immunol 2006, 18:220-225

24. Sharma MD, Baban B, Chandler P, Hou DY, Singh N, Yagita H, Azuma M, Blazar BR, Mellor AL, Munn DH: Plasmacytoid dendritic cells from mouse tumor-draining lymph nodes directly activate mature Tregs via indoleamine 2,3-dioxygenase. J Clin Invest 2007, 117:2570-2582

25. Fallarino F, Grohmann U, You S, McGrath BC, Cavener DR, Vacca C, Orabona C, Bianchi R, Belladonna ML, Volpi C, Santamaria P, Fioretti MC, Puccetti P: The combined effects of tryptophan starvation and tryptophan catabolites down-regulate $T$ cell receptor zeta-chain and induce a regulatory phenotype in naive T cells. J Immunol 2006, 176:6752-6761

26. Grohmann U, Orabona C, Fallarino F, Vacca C, Calcinaro F, Falorni A, Candeloro P, Belladonna ML, Bianchi R, Fioretti MC, Puccetti P: CTLA-4-Ig regulates tryptophan catabolism in vivo. Nat Immunol 2002, 3:1097-1101

27. Munn DH, Sharma MD, Lee JR, Jhaver KG, Johnson TS, Keskin DB, Marshall B, Chandler P, Antonia SJ, Burgess R, Slingluff CL Jr, Mellor AL: Potential regulatory function of human dendritic cells expressing indoleamine 2,3-dioxygenase. Science 2002, 297:1867-1870

28. Fallarino F, Grohmann U, Hwang KW, Orabona C, Vacca C, Bianchi R, Belladonna ML, Fioretti MC, Alegre ML, Puccetti P: Modulation of tryptophan catabolism by regulatory T cells. Nat Immunol 2003, $4: 1206-1212$

29. Soliman HH, Antonia S, Sullivan D, Vahanian N, Link C: Overcoming tumor antigen anergy in human malignancies using the novel indeolamine 2,3-dioxygenase (IDO) enzyme inhibitor, 1-methyl-D-tryptophan (1MT). J Clin Oncol 2009, 27:15s

30. Shankaran V, Ikeda H, Bruce AT, White JM, Swanson PE, Old LJ, Schreiber RD: IFNgamma and lymphocytes prevent primary tumour development and shape tumour immunogenicity. Nature 2001, 410:1107-1111

31. Coussens LM, Werb Z: Inflammation and cancer. Nature 2002, 420:860-867

32. Balkwill F, Charles KA, Mantovani A: Smoldering and polarized inflammation in the initiation and promotion of malignant disease. Cancer Cell 2005, 7:211-217

33. Gajewski TF: Failure at the effector phase: immune barriers at the level of the melanoma tumor microenvironment. Clin Cancer Res 2007, 13:5256-5261

34. Munn DH, Mellor AL: The tumor-draining lymph node as an immuneprivileged site. Immunol Rev 2006, 213:146-158

35. Sotomayor EM, Borrello I, Rattis FM, Cuenca AG, Abrams J, StaveleyO'Carroll K, Levitsky HI: Cross-presentation of tumor antigens by bone marrow-derived antigen-presenting cells is the dominant mechanism in the induction of T-cell tolerance during B-cell lymphoma progression. Blood 2001, 98:1070-1077

36. Yu P, Rowley DA, Fu YX, Schreiber $\mathrm{H}$ : The role of stroma in immune recognition and destruction of well-established solid tumors. Curr Opin Immunol 2006, 18:226-231

37. Zhang B, Bowerman NA, Salama JK, Schmidt H, Spiotto MT, Schietinger A, Yu P, Fu YX, Weichselbaum RR, Rowley DA, Kranz DM, Schreiber H: Induced sensitization of tumor stroma leads to eradication of established cancer by T cells. J Exp Med 2007, 204:49-55 
38. Willimsky G, Blankenstein T: Sporadic immunogenic tumours avoid destruction by inducing T-cell tolerance. Nature 2005, 437:141-146

39. Rygaard J, Povlsen CO: Heterotransplantation of a human malignant tumour to "Nude" mice. Acta Pathol Microbiol Scand 1969, $77: 758-760$

40. Kiessling R, Klein E, Pross $H$, Wigzell $H$ : "Natural" killer cells in the mouse. II. Cytoxic cells with specificity for mouse Moloney leukemia cells. Characteristics of the killer cell. Eur. J. Immunol 1975, 5:117-121
41. Grivennikov SI, Karin M: Inflammation and oncogenesis: a vicious connection. Curr Opin Genet Dev 2010, 20:62-71

42. Muller AJ, Scherle PA: Targeting the mechanisms of tumoral immune tolerance with small-molecule inhibitors. Nat Rev Cancer 2006, 6:613-625

43. Prendergast GC, Jaffee EM: Cancer immunologists and cancer biologists: why we didn't talk then but need to now. Cancer Res 2007, 67:3500-3504 